\title{
Comments on the high energy limit of bosonic open string theory
}

\author{
Chuan-Tsung Chan a, Pei-Ming Ho ${ }^{b}$, Jen-Chi Lee ${ }^{c}$, \\ Shunsuke Teraguchi ${ }^{\mathrm{d}, *}$, Yi Yang ${ }^{\mathrm{c}}$ \\ a Physics Division, National Center for Theoretical Sciences, Hsinchu, Taiwan \\ b Department of Physics, National Taiwan University, Taipei, Taiwan \\ c Department of Electrophysics, National Chiao-Tung University, Hsinchu, Taiwan \\ d Physics Division, National Center for Theoretical Sciences, Taipei, Taiwan
}

Received 25 March 2006; accepted 24 May 2006

Available online 12 June 2006

\begin{abstract}
In previous works, ratios among four-point scattering amplitudes at the leading order in the high-energy limit were derived for the bosonic open string theory. The derivation was based on Ward identities derived from the decoupling of zero-norm states and was purely algebraic. The only assumption of the derivation was that the momentum polarization can be approximated by the longitudinal polarization at high energies. In this paper, using the decoupling of spurious states, we reduce this assumption to a much weaker one which can be easily verified by simple power counting in most cases. For the special cases which are less obvious, we verify the new assumption for an example by saddle-point approximation. We also provide a new perspective to our previous results in terms of DDF states. In particular, we show that, by using DDF states, one can easily see that there is only one independent high energy scattering amplitude for each fixed mass level.
\end{abstract}

(c) 2006 Elsevier B.V. All rights reserved.

\section{Introduction}

One of the prominent characteristics of string theory is its huge spacetime gauge symmetry. In the usual world-sheet approach, this gauge symmetry is represented by zero-norm states in the

\footnotetext{
* Corresponding author.

E-mail addresses: ctchan@phys.cts.nthu.edu.tw (C.-T. Chan), pmho@ntu.edu.tw (P.-M. Ho), jcclee@cc.nctu.edu.tw (J.-C. Lee), teraguch@phys.ntu.edu.tw (S. Teraguchi), yiyang@mail.nctu.edu.tw (Y. Yang).
} 
spectrum and string interactions preserve this structure. It is reasonable to believe that, at least perturbatively, this huge gauge symmetry together with Lorentz symmetry govern the theory in flat spacetime.

In [1], it was conjectured that string theory also possesses a huge hidden symmetry which relates all string oscillation modes to one another, such that the S-matrix is determined by the scattering amplitudes among, say, tachyons for bosonic strings or dilatons for superstrings. Unfortunately, not much of this hidden symmetry has been understood.

In general, it is plausible that a hidden symmetry becomes manifest in the high-energy limit [2], where massive particles become effectively massless. The observation that only the above-mentioned gauge symmetry governs the theory suggests that the hidden symmetry is in some sense hidden in the gauge symmetry. ${ }^{1}$ If this is the case, taking the high-energy limit of the gauge symmetry might be a proper approach in order to obtain useful information about the conjectured hidden symmetry. Our previous works [3-5] were such efforts. For other attempts, see $[6,7]$.

In the papers $[3,4]$, we derived some linear relations among high-energy 4-point scattering amplitudes up to the third mass level by considering the high-energy limit of zero-norm states. Furthermore, in the paper [5], we generalized previous results to all mass levels. The strategy of our previous approach is the following. First, using the decoupling of zero-norm states from amplitudes, one obtains some linear relations (stringy Ward identities) among the amplitudes for unphysical states. ${ }^{2}$ Note that zero-norm states themselves do not relate physically inequivalent particle states, therefore we cannot obtain any physically meaningful relations at this point. However, after taking the high-energy limit of these relations, something special happens. Recall that 1-string states are characterized by a choice of polarizations in 26 dimensions. In this limit, momentum polarization and longitudinal polarization approach each other, and the transverse directions can be neglected except for the direction parallel to the scattering plane under consideration. Therefore, if we ignore the $1 / E^{2}$ effects, the system looks effectively two-dimensional ${ }^{3}$ and the above stringy Ward identities can relate amplitudes which now only involve two polarizations. Using these linear relations in the high-energy limit, one can obtain physically meaningful results. Remarkably, all the high-energy leading amplitudes for relevant physical states are completely solved and can be related to that of four tachyons.

However, there is a loophole in the above argument. While we only focused on the leading behavior of amplitudes, sometimes an amplitude vanishes accidentally at the presumed leading order, and the true leading order is lower than the naive expectation. In this case, we cannot fully justify the omission of the $1 / E^{2}$ effects (which are actually at the true leading order), and the replacement of the momentum polarization by the longitudinal polarization may not be a good approximation. As a simple example, using the notation in Eq. (1), the difference between $e^{P} \cdot \mathcal{T}$ and $e^{L} \cdot \mathcal{T}$ is at the leading order if $\mathcal{T} \propto e^{L}$ or $e^{P}$ although the difference between $e^{P}$ and $e^{L}$ is subleading in the high energy limit. Therefore, strictly speaking, our previous argument needs further justification. Roughly speaking, it was based on an assumption on the smoothness of the high-energy limit, as we explained in the paper [4]. Although this assumption is highly nontrivial from the viewpoint of ordinary field theories, the final results have been verified independently by

\footnotetext{
1 A possible connection between these symmetries is that the hidden symmetry is needed for the self-consistency of gauge-invariant interactions, as it is highly nontrivial for higher spin gauge theories to have self-consistent interactions.

2 Of course, "amplitude" for unphysical states is not well defined. They depend on the gauge-fixing prescription for world-sheet symmetry.

3 This is for the case of 4-point function.
} 
direct computations of four-point functions. The main aim of this paper is to fill in this possible loophole. Instead of starting with stringy Ward identities which are derived from the decoupling of zero-norm states, we utilize the decoupling of spurious states here. With a much weaker assumption, we can justify the omission of the $1 / E^{2}$ effects on the way to showing the irrelevance of other states, that is, the momentum polarization can be replaced by the longitudinal polarization at high energies. Besides this, the derivations of linear relations are quite similar to those in [5] based on the decoupling of high-energy zero-norm states. Though the new assumption for the proof of our results might seem always valid at first sight, if we consider multi-tensor scattering amplitudes, it is not always trivial, at least not until explicitly checked. For two-tensor cases, we shall show one example, and check that the assumption is valid. Based on this example, we argue that this assumption is valid for generic cases.

To summarize, we can now show with better rigor that the high-energy amplitudes of bosonic open strings are linearly related. Furthermore, only one independent function for high-energy amplitudes exists at each fixed mass level. This result suggests that effectively only one physical state survives in this high-energy limit at every mass level. In principle, using our results, one can always perform a change of the basis for physical states so that the ratios among the high-energy amplitudes become $1: 0: \cdots: 0$. Moreover, by properly choosing the gauge, we can explicitly see this remarkable fact. We shall also comment on this issue in this paper.

\section{Rederivation of the general formula and justification of the replacement of $P$ by $L$}

\subsection{The high-energy limit}

Before detailing the proof of our result, we briefly summarize our procedure of taking the high-energy limit. See [5] for more details. We only consider 4-point scattering amplitudes of open strings in bosonic string theory for simplicity. These 4-point amplitudes depend on the center-of-mass energy $E_{\mathrm{cm}}$, the scattering angle $\phi$ and the choices of four oscillating modes of strings. We take the limit ${ }^{4}$ of $E_{\mathrm{cm}}$ going to infinity with the scattering angle $\phi$ fixed. Three of the particles are fixed and the mass level of the last particle is a fixed integer $n$. We will use the terminology "family" to represents a class of particles which are at the same mass level $n$. Our question is how the leading behavior of the amplitudes will change when we replace one of the particles by another particle in the same family. Now we will focus on the string state of the last particle. To specify the polarizations of this state, we use the following basis for 26 dimensions

$$
\begin{aligned}
e^{P} & =\frac{1}{m}\left(\sqrt{p^{2}+m^{2}}, p, 0, \ldots, 0\right), \quad e^{L}=\frac{1}{m}\left(p, \sqrt{p^{2}+m^{2}}, 0, \ldots, 0\right), \\
e^{T_{i}} & =(0,0, \ldots, 1, \ldots)
\end{aligned}
$$

where $m$ is the mass of the state at issue given by $\sqrt{2(n-1)}$. It will be convenient to introduce two more vectors

$$
\begin{aligned}
& e^{T}=(0,0, \ldots, 0,1), \\
& e^{(L-P)}=\frac{p-\sqrt{p^{2}+m^{2}}}{m}(1,1,0, \ldots, 0) \simeq-\frac{m}{2 p}(1,1,0, \ldots, 0),
\end{aligned}
$$

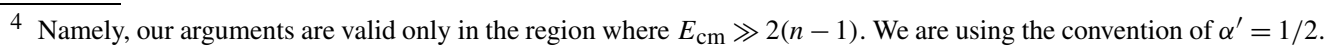


where $e^{T}$ is the transverse vector which is parallel to the scattering plane. ${ }^{5}$ From now on, we reserve the notation $e^{T_{i}}$ for the transverse polarizations which are perpendicular to the scattering plane. $e^{(L-P)}$ is a null vector which is defined by the difference between $e^{P}$ and $e^{L}$. The inner product of a vector $V^{\mu}$ with these unit vectors $e_{\mu}^{A}\left(A=P, L, T, T_{i}\right.$ or $\left.(L-P)\right)$ will be denoted by $V^{A}$.

\subsection{Definitions and assumption}

In the rest of this section, we give a better proof of our main result in [5]. This will be achieved in several steps. First, we will assign naive energy dimensions to each oscillation modes based on concrete calculation of scattering amplitudes. Second, we will have to make a minor assumption about how 4-point functions really scale with energy $E$ in the high-energy limit, namely, we assume that, for some class of states, we can trust the power counting of the naive energy dimensions. This assumption can sometimes be easily checked using the saddle-point approximation. From the decoupling of spurious states and this assumption, we can tell which states should not contribute to scattering amplitudes in the high-energy limit. This information will allow us to freely replace the polarization $P$ by $L$ in the linear relations of 4-point functions obtained from the decoupling of spurious states. The last step is to show that these linear relations lead to our earlier result [5], which is the explicit expression of ratios among scattering amplitudes.

As we just mentioned, we use the decoupling of spurious states from physical states to rederive our previous result in a rigorous manner. We only need two Virasoro operators

$$
\begin{aligned}
& L_{-1}=\frac{1}{2} \sum_{n \in \mathbb{Z}} \alpha_{-1+n} \cdot \alpha_{-n}=\hat{m} \alpha_{-1}^{P}+\alpha_{-2} \cdot \alpha_{1}+\cdots, \\
& L_{-2}=\frac{1}{2} \sum_{n \in \mathbb{Z}} \alpha_{-2+n} \cdot \alpha_{-n}=\frac{1}{2} \alpha_{-1} \cdot \alpha_{-1}+\hat{m} \alpha_{-2}^{P}+\alpha_{-3} \cdot \alpha_{1}+\cdots,
\end{aligned}
$$

to generate all spurious states. Each oscillator $\alpha_{-m}^{A}$ corresponds to a factor of $\frac{1}{(m-1) !} \partial^{m} X^{A}$ in the vertex operator. The operator $\partial^{m} X^{\mu}$ can contract with the exponent $i k \cdot X$ of another vertex operator in the correlation function to produce a factor of $k^{\mu}$. This is the leading order contribution of the factor $\partial^{m} X^{\mu}$ to the correlation function, and it scales like $E^{1}$ at high energies. So we assign a dimension 1 to $\partial^{m} X^{\mu}$. Similarly we assign dimensions to the polarization vectors. Combining the dimensions of $X^{\mu}$ and $e_{\mu}^{A}$, we associate a naive dimension to every oscillator

$$
\alpha_{-m}^{P} \rightarrow 2, \quad \alpha_{-m}^{L} \rightarrow 2, \quad \alpha_{-m}^{T} \rightarrow 1, \quad \alpha_{-m}^{T_{i}} \rightarrow 0, \quad \alpha_{-m}^{(L-P)} \rightarrow 0 .
$$

However, terms at the naive leading order may happen to cancel (this happens whenever $\partial X^{P}$ or $\partial X^{L}$ is involved [3-5]) and the true leading order may be lower (but never higher).

Another notion that will be helpful is the naive dimension of a state, which is the sum of the naive dimensions of all creation operators needed to create the state from vacuum. We symbolically represent a generic state at level $n$ (with mass $\hat{m}=\sqrt{2(n-1)}$ ) and naive dimension $d$ as $|n, d\rangle$. As an example of using this notation, we have

$$
L_{-1}|n-1, d\rangle=\hat{m} \alpha_{-1}^{P}|n-1, d\rangle+|n, d\rangle .
$$

Let us now state our assumption.

\footnotetext{
5 In this paper, we set the scattering plane on the $X^{0}-X^{25}$ plane.
} 
Assumption. In the following we will assume that the true energy order of the amplitude for the state

$$
|n\rangle=\overbrace{\alpha_{-1}^{T} \cdots \alpha_{-1}^{T}}^{n}|0\rangle
$$

(with other particles fixed) is greater than the amplitude for any state $|n, d\rangle$ whose naive dimension $d$ is less than $n$.

Note that this is the only input of our derivation apart from the decoupling of spurious states. Using the saddle-point method, one can immediately conclude that the assumption is correct for 4-point functions when the prefactors of the other 3 vertices do not contain $\partial X^{L}$. Therefore the assumption only needs to be checked when $\partial X^{L}$ does appear in one or more of the other 3 vertices. We do not have a rigorous proof, but the assumption holds for all examples we have checked. (See Section 4.)

In fact, our algebraic proof can be applied to generic $N$-point functions. For $N$-point functions, we fix $(N-1)$ vertices such that the amplitude is not suppressed when the varying vertex is chosen to be $|n\rangle$. The same procedure given below will allow us to find the ratios between the $N$-point function for $|n\rangle$ and certain other choices of states at the same level. However, unique solution for all possible choices of vertices can be derived only for 4-point functions because in other cases we cannot ignore all other transverse polarizations.

\subsection{Proof of irrelevance}

The first step is to find those states which are subleading compared to $|n\rangle$, so that we can ignore them later. We first show that a state is subleading if the total number of $\alpha_{-m}^{P}$ and $\alpha_{-m}^{L}$ is odd.

\subsubsection{Irrelevance of the states with only one $\alpha_{-1}^{P}$}

To begin, we prove that states involving a single factor of $\alpha_{-1}^{P}$ are subleading. Consider a class of spurious states generated by $L_{-1}$

$$
L_{-1}|n-1, n-1\rangle=\hat{m} \alpha_{-1}^{P}|n-1, n-1\rangle+\underbrace{|n, n-1\rangle}_{\text {irrelevant }} .
$$

Due to the assumption (7) above, the state $|n, n-1\rangle$ is not at the leading order and can be ignored. The decoupling of the spurious state implies that

$$
\alpha_{-1}^{P}|n-1, n-1\rangle \rightarrow \text { irrelevant },
$$

though the naive dimension of this state is $n+1$.

\subsubsection{Irrelevance of the states with three $\alpha_{-1}^{P}$}

The next set of spurious states we consider is

$$
\begin{aligned}
& L_{-1} \alpha_{-1}^{P}|n-2, n-3\rangle \\
& \quad=\hat{m} \alpha_{-1}^{P} \alpha_{-1}^{P}|n-2, n-3\rangle+\underbrace{\alpha_{-2}^{P}|n-2, n-3\rangle}_{\text {irrelevant }}+\underbrace{\alpha_{-1}^{P}|n-1, n-3\rangle}_{\text {irrelevant }} .
\end{aligned}
$$


The last two terms are of naive leading order $(n-1)$, implying that the first term on the righthand side is decoupled in the high energy limit, despite the fact that it has a naive dimension of $(n+1)$. Similarly, we have

$$
\begin{aligned}
& L_{-1} \alpha_{-1}^{P} \alpha_{-1}^{P}|n-3, n-3\rangle \\
& \quad=\hat{m} \alpha_{-1}^{P} \alpha_{-1}^{P} \alpha_{-1}^{P}|n-3, n-3\rangle+2 \underbrace{\alpha_{-1}^{P} \alpha_{-2}^{P}|n-3, n-3\rangle}_{\text {irrelevant } \leftarrow \text { (9) }}+\underbrace{\alpha_{-1}^{P} \alpha_{-1}^{P}|n-2, n-3\rangle}_{\text {irrelevant } \leftarrow \text { (10) }},
\end{aligned}
$$

and we conclude that both class of states are irrelevant at high energies

$$
\alpha_{-1}^{P} \alpha_{-1}^{P}|n-2, n-3\rangle, \quad \text { and } \quad \alpha_{-1}^{P} \alpha_{-1}^{P} \alpha_{-1}^{P}|n-3, n-3\rangle \rightarrow \text { irrelevant. }
$$

\subsubsection{Irrelevance of the states with odd numbers of $\alpha_{-1}^{P}$ and $\alpha_{-1}^{L}$}

The previous result (12) allows us to use mathematical induction. We need to prove that if both

$$
\begin{aligned}
& \overbrace{\alpha_{-1}^{P} \cdots \alpha_{-1}^{P}}^{2 k-2}|n-2 k+2, n-2 k+1\rangle, \quad \text { and } \\
& \overbrace{\alpha_{-1}^{P} \cdots \alpha_{-1}^{P}}^{2 k-1}|n-2 k+1, n-2 k+1\rangle \rightarrow \text { irrelevant, }
\end{aligned}
$$

then

$$
\begin{aligned}
& \overbrace{\alpha_{-1}^{P} \cdots \alpha_{-1}^{P}}^{2 k}|n-2 k, n-2 k-1\rangle, \quad \text { and } \\
& \overbrace{\alpha_{-1}^{P} \cdots \alpha_{-1}^{P}}^{2 k+1}|n-2 k-1, n-2 k-1\rangle \rightarrow \text { irrelevant. }
\end{aligned}
$$

The proof is consisted of computing the following two types of spurious states:

$$
\begin{aligned}
& L_{-1} \overbrace{\alpha_{-1}^{P} \cdots \alpha_{-1}^{P}}^{2 k-1}|n-2 k, n-2 k-1\rangle \\
&=\hat{m} \overbrace{\alpha_{-1}^{P} \cdots \alpha_{-1}^{P}}^{2 k}|n-2 k, n-2 k-1\rangle+(2 k-1) \underbrace{\overbrace{\alpha_{-1}^{P} \cdots \alpha_{-1}^{P}}^{2 k-2} \alpha_{-2}^{P}|n-2 k, n-2 k-1\rangle}_{\text {irrelevant } \leftarrow(13)} \\
&+\overbrace{\underbrace{\alpha_{-1}^{P} \cdots \alpha_{-1}^{P}}_{\text {irrelevant } \leftarrow(13)}|n-2 k+1, n-2 k-1\rangle}^{2 k-1},
\end{aligned}
$$

and

$$
\begin{aligned}
& L_{-1} \overbrace{\alpha_{-1}^{P} \cdots \alpha_{-1}^{P}}^{2 k}|n-2 k-1, n-2 k-1\rangle \\
& =\hat{m} \overbrace{\alpha_{-1}^{P} \cdots \alpha_{-1}^{P}}^{2 k+1}|n-2 k-1, n-2 k-1\rangle
\end{aligned}
$$




$$
\begin{aligned}
& +2 k \underbrace{\overbrace{\alpha_{-1}^{P} \cdots \alpha_{-1}^{P}}^{2 k-1} \alpha_{-2}^{P}|n-2 k-1, n-2 k-1\rangle}_{\text {irrelevant } \leftarrow(13)} \\
& +\underbrace{\overbrace{\alpha_{-1}^{P} \cdots \alpha_{-1}^{P}}^{2 k}|n-2 k, n-2 k-1\rangle}_{\text {irrelevant } \leftarrow(15)} .
\end{aligned}
$$

Therefore, both type of states

$$
\overbrace{\alpha_{-1}^{P} \cdots \alpha_{-1}^{P}}^{2 k}|n-2 k, n-2 k-1\rangle, \quad \text { and } \overbrace{\alpha_{-1}^{P} \cdots \alpha_{-1}^{P}}^{2 k+1}|n-2 k-1, n-2 k-1\rangle,
$$

can be ignored. Using the identity

$$
\alpha_{-1}^{L}=\alpha_{-1}^{P}+\alpha_{-1}^{(L-P)},
$$

we conclude that

$$
\begin{aligned}
& \overbrace{\alpha_{-1}^{P} \cdots \alpha_{-1}^{P} \alpha_{-1}^{L} \cdots \alpha_{-1}^{L}}^{2 k}|n-2 k, n-2 k-1\rangle, \quad \text { and } \\
& \overbrace{\alpha_{-1}^{P} \cdots \alpha_{-1}^{P} \alpha_{-1}^{L} \cdots \alpha_{-1}^{L}}^{2 k+1}|n-2 k-1, n-2 k-1\rangle,
\end{aligned}
$$

are also irrelevant, because the naive dimension of $\alpha_{-1}^{(L-P)}$ is zero.

\subsection{Linear relations}

In this section we rederive the linear relations among 4-point functions obtained in [5].

So far, we have shown that if a state is not of this form,

$$
\overbrace{\alpha_{-1}^{P} \cdots \alpha_{-1}^{P} \alpha_{-1}^{L} \cdots \alpha_{-1}^{L}}^{2 k}|n-2 k, n-2 k\rangle,
$$

then the state is irrelevant. Furthermore, it indicates that a combination

$$
\begin{aligned}
& \left(\alpha_{-1}^{L} \alpha_{-1}^{L}-\alpha_{-1}^{P} \alpha_{-1}^{P}\right) \overbrace{\alpha_{-1}^{P} \cdots \alpha_{-1}^{P} \alpha_{-1}^{L} \cdots \alpha_{-1}^{L}}^{2 k}|n-2 k-2, n-2 k-2\rangle \\
& \quad=\left(2 \alpha_{-1}^{P} \alpha_{-1}^{(L-P)}+\alpha_{-1}^{(L-P)} \alpha_{-1}^{(L-P)}\right) \overbrace{\alpha_{-1}^{P} \cdots \alpha_{-1}^{P} \alpha_{-1}^{L} \cdots \alpha_{-1}^{L}}^{2 k}|n-2 k-2, n-2 k-2\rangle,
\end{aligned}
$$

is also irrelevant. By definition, $\overbrace{\alpha_{-1}^{T} \cdots \alpha_{-1}^{T}}^{n}|0\rangle$ is relevant. Now we derive relations among the above type of states and see that all of them are relevant. Hereafter, we use the notation

$$
\mathcal{T}^{(n, 2 m, q)}
$$

to represent scattering amplitudes corresponding to the states

$$
|n, 2 m, q\rangle \equiv\left(\alpha_{-1}^{T}\right)^{n-2 m-2 q}\left(\alpha_{-1}^{P}\right)^{2 m}\left(\alpha_{-2}^{P}\right)^{q}|0 ; k\rangle .
$$




\subsubsection{Relation 1}

Consider the spurious state

$$
\begin{aligned}
& L_{-2} \overbrace{\alpha_{-1}^{T} \cdots \alpha_{-1}^{T}}^{n-2}|0\rangle \\
& =(\frac{1}{2}(\underbrace{\alpha_{-1}^{T} \alpha_{-1}^{T}}_{\text {relevant }}+\underbrace{\alpha_{-1}^{L} \alpha_{-1}^{L}-\alpha_{-1}^{P} \alpha_{-1}^{P}}_{\text {irrelevant }})+\hat{m} \alpha_{-2}^{P}+\underbrace{\alpha_{-3} \cdot \alpha_{1}+\cdots}_{\text {irrelevant }}) \overbrace{\alpha_{-1}^{T} \cdots \alpha_{-1}^{T}}^{n-2}|0\rangle .
\end{aligned}
$$

We see that $\overbrace{\alpha_{-1}^{T} \cdots \alpha_{-1}^{T}}^{n-2} \alpha_{-2}^{P}|0\rangle$ is relevant and

$$
\mathcal{T}^{(n, 0,1)}=-\frac{1}{2 \hat{m}} \mathcal{T}^{(n, 0,0)} \text {. }
$$

Using mathematical induction, we find that $\overbrace{\alpha_{-1}^{T} \cdots \alpha_{-1}^{T}}^{n-2 q} \overbrace{\alpha_{-2}^{P} \cdots \alpha_{-2}^{P}}^{q}|0\rangle$ are relevant and

$$
\mathcal{T}^{(n, 0, q)}=\left(\frac{-1}{2 \hat{m}}\right)^{q} \mathcal{T}^{(n, 0,0)}
$$

\subsubsection{Relation 2}

Consider another class of spurious states

$$
\begin{aligned}
& L_{-1} \overbrace{\alpha_{-1}^{T} \cdots \alpha_{-1}^{T}}^{n-2 q-2} \alpha_{-1}^{P} \overbrace{\alpha_{-2}^{P} \cdots \alpha_{-2}^{P}}^{q}|0\rangle \\
& =(\hat{m} \alpha_{-1}^{P}+\alpha_{-2} \cdot \alpha_{1}+\underbrace{\alpha_{-3} \cdot \alpha_{2}+\cdots}_{\text {irrelevant }}) \overbrace{\alpha_{-1}^{T} \cdots \alpha_{-1}^{T}}^{n-2 q-2} \alpha_{-1}^{P} \overbrace{\alpha_{-2}^{P} \cdots \alpha_{-2}^{P}}^{q}|0\rangle \\
& =\hat{m} \overbrace{\alpha_{-1}^{T} \cdots \alpha_{-1}^{T}}^{n-2 q-2} \alpha_{-1}^{P} \alpha_{-1}^{P} \overbrace{\alpha_{-2}^{P} \cdots \alpha_{-2}^{P}}^{q}|0\rangle+\overbrace{\underbrace{\overbrace{\alpha_{-1}^{T} \cdots \alpha_{-1}^{T}}^{n-2 q-2} \overbrace{\alpha_{-2}^{P} \cdots \alpha_{-2}^{P}}^{q}|0\rangle}_{\text {relevant }}+\text { irrelevant. }}^{q+1}
\end{aligned}
$$

It shows that $\overbrace{\alpha_{-1}^{T} \cdots \alpha_{-1}^{T}}^{n-2 q-2} \alpha_{-1}^{P} \alpha_{-1}^{P} \overbrace{\alpha_{-2}^{P} \cdots \alpha_{-2}^{P}}^{q}|0\rangle$ is relevant and

$$
\mathcal{T}^{(n, 2, q)}=-\frac{1}{\hat{m}} \mathcal{T}^{(n, 0, q+1)} \text {. }
$$

Using mathematical induction again, we find that $\overbrace{\alpha_{-1}^{T} \cdots \alpha_{-1}^{T}}^{n-2 m-2 q} \overbrace{\alpha_{-1}^{P} \cdots \alpha_{-1}^{P}}^{2 m} \overbrace{\alpha_{-2}^{P} \cdots \alpha_{-2}^{P}}^{q}|0\rangle$ are relevant and

$$
\mathcal{T}^{(n, 2 m, q)}=\left(\frac{-(2 m-1)}{\hat{m}}\right) \cdots\left(\frac{-3}{\hat{m}}\right)\left(\frac{-1}{\hat{m}}\right) \mathcal{T}^{(n, 0, q+m)} .
$$




\subsection{Final result}

Because we know the irrelevance of other states, the flipping of $P$ to $L$ is justified for relevant states. Therefore all relevant states take the form

$$
\overbrace{\alpha_{-1}^{T} \cdots \alpha_{-1}^{T}}^{n-2 m-2 q} \overbrace{\alpha_{-1}^{P} \cdots \alpha_{-1}^{P} \alpha_{-1}^{L} \cdots \alpha_{-1}^{L}}^{2 m} \overbrace{\alpha_{-2}^{P} \cdots \alpha_{-2}^{P} \alpha_{-2}^{L} \cdots \alpha_{-2}^{L}}^{q}|0\rangle,
$$

and their amplitudes are related to that of the reference state $|n\rangle=\overbrace{\alpha_{-1}^{T} \cdots \alpha_{-1}^{T}}^{n}|0\rangle$ by

$$
\mathcal{T}^{(n, 2 m, q)}=\left(\frac{-(2 m-1)}{\hat{m}}\right) \cdots\left(\frac{-3}{\hat{m}}\right)\left(\frac{-1}{\hat{m}}\right)\left(\frac{-1}{2 \hat{m}}\right)^{m+q} \mathcal{T}^{(n, 0,0)} .
$$

This is finally our main result of the previous paper [5].

\section{Choice of gauge}

In the last section, we have proved, under the assumption (7), that, in the high energy limit, all 4-point correlation functions are linearly related and remarkably there is a unique function for every family. It suggests that there exists such a choice of basis for physical particles, where only one particle in the same family gives a non-zero scattering amplitude. Though such a basis could in principle be given in any gauge, we found that there exists a suitable gauge where one immediately realizes that only one physical particle survives in the high energy limit for every mass level.

This gauge is naturally spanned by DDF positive norm states [8]. DDF positive norm states are created by acting DDF operators

$$
A_{n}^{i}=\frac{1}{2 \pi} \int_{0}^{2 \pi} \dot{X}^{i}(\tau) e^{i n X^{+}(\tau)} d \tau, \quad i=1, \ldots, 24
$$

on the tachyonic ground state $\left|0, p_{0}\right\rangle$, where the tachyonic momentum is chosen as $p_{0}^{\mu}=$ $(0, \ldots, 0, \sqrt{2})$. It is well known that such states span the whole spectrum of physical positive norm states

$$
A_{-n_{1}}^{i_{1}} A_{-n_{2}}^{i_{2}} \cdots A_{-n_{m}}^{i_{m}}\left|0, p_{0}\right\rangle
$$

in a frame where the momentum of these states takes the form of $p^{\mu}=p_{0}^{\mu}-N k_{0}^{\mu}$, with $k_{0}=$ $(-1,0, \ldots, 0,1) / \sqrt{2}$ and $N$ representing the level of states. Since this construction of physical positive norm states naturally picks up a gauge, we simply call this gauge the DDF gauge. This gauge is characterized by the condition

$$
\left.k_{0} \cdot \alpha_{n} \mid \text { physical state }\right\rangle=0,
$$

for $n>0$. This condition guarantees that, in this gauge, physical states contain only oscillators whose polarizations are perpendicular to $k_{0}$. (Note that $k_{0}$ is perpendicular to itself because it is a null vector.) 
After short calculation, we can rewrite the DDF states in terms of the usual Fock space representation in any Lorentz flame. For example, for the 1 st massive particles $\left(m^{2}=2\right)$, we have

$$
\begin{aligned}
& A_{-1}^{i} A_{-1}^{j}\left|0, p_{0}\right\rangle \rightarrow\left(\alpha_{-1}^{i} \alpha_{-1}^{j}+\delta^{i j}\left(-\frac{1}{2 \sqrt{2}} \alpha_{-2}^{(L-P)}+\frac{1}{4} \alpha_{-1}^{(L-P)} \alpha_{-1}^{(L-P)}\right)\right)|0, p\rangle, \\
& A_{-2}^{i}\left|0, p_{0}\right\rangle \rightarrow\left(\alpha_{-2}^{i}-\sqrt{2} \alpha_{-1}^{i} \alpha_{-1}^{(L-P)}\right)|0, p\rangle,
\end{aligned}
$$

and for the 2 nd massive particles $\left(m^{2}=4\right)$

$$
\begin{aligned}
A_{-1}^{i} & A_{-1}^{j} A_{-1}^{k}\left|0, p_{0}\right\rangle \\
\rightarrow & \left(\alpha_{-1}^{i} \alpha_{-1}^{j} \alpha_{-1}^{k}\right. \\
& \left.\quad+\left(\delta^{i j} \alpha_{-1}^{k}+\delta^{k i} \alpha_{-1}^{j}+\delta^{j k} \alpha_{-1}^{i}\right)\left(-\frac{1}{4} \alpha_{-2}^{(L-P)}+\frac{1}{8} \alpha_{-1}^{(L-P)} \alpha_{-1}^{(L-P)}\right)\right)|0, p\rangle, \\
& A_{-2}^{i} A_{-1}^{j}\left|0, p_{0}\right\rangle \\
& \rightarrow\left(\alpha_{-2}^{i} \alpha_{-1}^{j}-\alpha_{-1}^{i} \alpha_{-1}^{j} \alpha_{-1}^{(L-P)}\right. \\
& \left.\quad+\delta^{i j}\left(-\frac{1}{3} \alpha_{-3}^{(L-P)}+\frac{1}{2} \alpha_{-2}^{(L-P)} \alpha_{-1}^{(L-P)}-\frac{1}{6} \alpha_{-1}^{(L-P)} \alpha_{-1}^{(L-P)} \alpha_{-1}^{(L-P)}\right)\right)|0, p\rangle, \\
A_{-3}^{i}\left|0, p_{0}\right\rangle & \quad\left(\alpha_{-3}^{i}-\frac{3}{2} \alpha_{-2}^{i} \alpha_{-1}^{(L-P)}+\alpha_{-1}^{i}\left(-\frac{3}{4} \alpha_{-2}^{(L-P)}-\frac{9}{8} \alpha_{-1}^{(L-P)} \alpha_{-1}^{(L-P)}\right)\right)|0, p\rangle .
\end{aligned}
$$

The polarization $e^{(L-P)}$ of oscillator $\alpha_{-n}^{(L-P)}$ emerges as a covariantized version of the null vector $k_{0}$. This explicit expressions of the physical positive norm states and our previous counting rules of naive dimension (5) imply that only the particles (35) and (37) with $i, j, k=T$ are relevant in the family at mass levels $m^{2}=2$ and $m^{2}=4$, respectively. Actually, this kind of structure is generic in this gauge. Noticing that the DDF operator (32) is at most linear in oscillators $\alpha_{-n}^{i}$, and the naive dimension of $\alpha_{-n}^{(L-P)}$ is 0 , we should assign naive dimension 1 to $A_{-n}^{T}$ and 0 to others. Therefore, we can conclude that the unique state

$$
A_{-1}^{T} \cdots A_{-1}^{T}\left|0, p_{0}\right\rangle
$$

constructed by only one operator, $A_{-1}^{T}$, survives in the high energy limit, under the same assumption as we have made in the previous section.

The existence of the DDF gauge where only the transverse and the $(L-P)$-polarization are needed is a direct implication of the fact that DDF states include all inequivalent physical states (up to Lorentz transformations). In this gauge we can easily see that the ratio of high energy amplitude is simply $1: 0: \cdots: 0$, while in other choices of gauge (and thus different choices of basis), they might be different. For instance, in the gauge where we use only the transverse and longitudinal polarizations, the ratio is $8: 1:-1:-1$ for $m^{2}=4$ (for the basis chosen in [3]).

\section{Validity of the assumption}

In the previous sections, we have studied behaviors of stringy amplitudes in the high-energy limit, based on the assumption made in Section 2. In this section, we shall check the valid- 
ity of the assumption (7). At first sight, one might think that the validity of the assumption is rather trivial. If there is no suppression of the energy orders of the high-energy amplitudes with the particle $|n\rangle$, we can regard their naive dimensions as the true ones, hence the assumption is valid. But we know that, in some situations, the high-energy amplitudes are suppressed and their true energy orders become less than the naive ones. Typically, such a suppression occurs when the other vertices contain $\alpha_{-1}^{P}$ or $\alpha_{-1}^{L}$. Originally, Wick-contracted terms between tensor parts of different vertex operators give subleading contributions compared to those from Wickcontracted terms between tensors and exponents. However, if the (naive) leading contributions turn out to cancel and the amplitudes are suppressed, it is very likely that the true leading contributions to a multi-tensor amplitude will be given by these tensor-tensor contraction terms. In that situation, the naive energy orders of amplitudes by dimensional analysis, which is based on Wick-contractions between tensors and exponents, might fail. Therefore, we need to check the validity of our assumption in order to confirm the main results (20), (31) and (40).

To be specific, we shall explicitly check the assumption in a representative example, where the four-point function consists of two spin-two tensors (at mass level $m^{2}=2$ ) and two tachyons. In particular, we shall fix $V_{1}$ as a physical state which consists $\alpha_{-1}^{L}$, and we can expect that the amplitude gets suppressed. We shall calculate the following amplitude

$$
\mathcal{T}=\int \prod_{i=1}^{4} d x_{i}\left\langle V_{1} V_{2} V_{3} V_{4}\right\rangle,
$$

where

$$
V_{1} \equiv \partial X^{T_{1}} \partial X^{L_{1}} e^{i k_{1} X},
$$

and $V_{3}, V_{4}$ are tachyon vertices. For $V_{2}$, we consider the following three cases:

Case 1: $\quad V_{2}=\partial X^{T_{2}} \partial X^{T_{2}} e^{i k_{2} X}$,

Case 2: $\quad V_{2}=\partial X^{T_{2}} \partial X^{\left(L_{2}-P_{2}\right)} e^{i k_{2} X}$,

Case 3: $\quad V_{2}=\partial X^{\left(L_{2}-P_{2}\right)} \partial X^{\left(L_{2}-P_{2}\right)} e^{i k_{2} X}$.

Notice that by our energy-counting rule (5), the four-point amplitudes associated with the three cases should have naive dimensions five, four and three, respectively. While it is conceivable that the true leading energy order for the first case, due to the presence of $\partial X^{L_{1}}$, should be no greater than three; it is not trivial to see whether the true leading orders of the second and the third cases are really less than that of the first case such that our assumption (7) can be justified. For this reason, we need to perform a sample calculation, based on the saddle-point method. ${ }^{6}$

The amplitude with two spin-two tensors and two tachyons is given by ${ }^{7}$

$$
\int_{-\infty}^{\infty} d x u(x) e^{-K f(x)},
$$

where

$$
K=-k_{1} \cdot k_{2}, \quad \tau=-\frac{k_{2} \cdot k_{3}}{k_{1} \cdot k_{2}},
$$

\footnotetext{
6 For details of the saddle-point method, see Section 5 of [5].

7 We have employed the standard $S L(2, R)$ gauge fixing, $x_{1}=0, x_{3}=1, x_{4}=\infty$, such that $k_{14}, k_{24}$ and $k_{34}$ do not appear in this discussion.
} 


$$
f(x)=\ln x-\tau \ln (1-x),
$$

and the function $u(x)$ consists of three contributions with different energy orders. It is convenient to make the following decomposition,

$$
u(x) \equiv u_{\mathrm{I}}(x)+u_{\mathrm{II}}(x)+u_{\mathrm{III}}(x),
$$

and

$$
\begin{aligned}
u_{\mathrm{I}}(x) \equiv & \left(e^{T_{1}} \cdot k_{23}\right)\left(e^{L_{1}} \cdot k_{23}\right)\left(e^{A} \cdot k_{13}\right)\left(e^{B} \cdot k_{13}\right), \\
u_{\mathrm{II}}(x) \equiv & -\frac{1}{x^{2}}\left[\left(e^{T_{1}} \cdot e^{A}\right)\left(e^{L_{1}} \cdot k_{23}\right)\left(e^{B} \cdot k_{13}\right)+\left(e^{T_{1}} \cdot e^{B}\right)\left(e^{L_{1}} \cdot k_{23}\right)\left(e^{A} \cdot k_{13}\right)\right. \\
& \left.+\left(e^{L_{1}} \cdot e^{A}\right)\left(e^{T_{1}} \cdot k_{23}\right)\left(e^{B} \cdot k_{13}\right)+\left(e^{L_{1}} \cdot e^{B}\right)\left(e^{T_{1}} \cdot k_{23}\right)\left(e^{A} \cdot k_{13}\right)\right], \\
u_{\mathrm{III}}(x) \equiv & \frac{1}{x^{4}}\left[\left(e^{T_{1}} \cdot e^{A}\right)\left(e^{L_{1}} \cdot e^{B}\right)+\left(e^{T_{1}} \cdot e^{B}\right)\left(e^{L_{1}} \cdot e^{A}\right)\right],
\end{aligned}
$$

where $e_{\mu}^{A} e_{\nu}^{B}$ is the polarization tensor of the second particle and

$$
\begin{aligned}
& k_{23} \equiv \frac{k_{2}}{x}+k_{3}, \\
& k_{13} \equiv \frac{k_{1}}{x}-\frac{k_{3}}{1-x},
\end{aligned}
$$

are linear combinations of the momenta, which come from the Wick-contraction with $e^{i k \cdot X} \mathrm{~s}$. The function $u_{\mathrm{I}}(x)$ is the part of $u(x)$ which does not contain terms from tensor-tensor contraction. $u_{\mathrm{II}}(x)$ and $u_{\mathrm{III}}$ come from the terms with one tensor-tensor contraction and with two tensor-tensor contractions, respectively. Note that, when we use the saddle-point approximation and substitute its saddle-point value $x_{0}=1 /(1-\tau)$ for moduli parameter $x$, the inner products $e^{L_{1}} \cdot k_{23}$ and $e^{L_{2}} \cdot k_{13}$ get suppressed and their true energy orders become one. In case 1 , in the zeroth-order contribution of the saddle-point approximation, we can see the expected suppression for both $u_{\mathrm{I}}\left(x_{0}\right)$ and $u_{\mathrm{II}}\left(x_{0}\right)$, and $u_{\mathrm{III}}\left(x_{0}\right)$ is identically zero because the polarizations are orthogonal to $e^{L_{1}}$. Then, we conclude that the true energy order of the amplitude is really three, after checking that there is no further suppression between the zeroth-order contribution of $u_{\mathrm{I}}(x)$ and the first-order contribution of $u_{\mathrm{I}}(x)$ in the saddle-point approximation. In view of this, for the validity of the assumption, we need to make sure that the true energy orders of cases 2 and 3 are really less than three. Indeed, due to the existence of $e^{L_{1}}$ in $V_{1}, u_{\mathrm{I}}(x)$ for both cases 2 and 3 also get suppressed and have energy order two and one, respectively. In general, $u_{\mathrm{I}}(x)$ always shares a common pattern of suppression for different choices of $V_{2}$, because it is of a factorized form. Furthermore, for cases 2 and 3, naive energy orders (hence, true energy orders) of $u_{\mathrm{II}}(x)$ and $u_{\mathrm{III}}(x)$ are no greater than two. ${ }^{8}$ Thus, in this example, our assumption is valid.

Now we can apply our results to this example and check one of their consequences. For example, if we consider the vertex

$$
\text { Case 4: } \quad V_{2}=\partial X^{T_{2}} \partial X^{L_{2}} e^{i k_{2} X},
$$

according to our results, this amplitude should have true energy order less than that of case 1, namely, three. At first sight, it does not seem to happen because the true energy order of $u_{\mathrm{II}}\left(x_{0}\right)$

\footnotetext{
8 Actually, they do not get suppressed in this example.
} 
Table 1

\begin{tabular}{llll}
\hline & $u_{\mathrm{I}}(x)$ & $u_{\mathrm{II}}(x)$ & $u_{\mathrm{III}}(x)$ \\
\hline Case 1 & $E^{5} \rightarrow E^{3}$ & $E^{3} \rightarrow E^{1}$ & 0 \\
Case 2 & $E^{4} \rightarrow E^{2}$ & $E^{2}$ & $E^{0}$ \\
Case 3 & $E^{3} \rightarrow E^{1}$ & $E^{1}$ & $E^{-1}$ \\
Case 4 & $E^{6} \rightarrow E^{4}$ & $E^{4}$ & $E^{2}$ \\
& & $E^{4}+E^{4} \rightarrow E^{2}$ & \\
\hline
\end{tabular}

is actually four. However, explicit calculation shows that the first-order contribution of $u_{\mathrm{I}}(x)$ in the saddle-point approximation (miraculously) cancels the leading contribution of $u_{\mathrm{II}}\left(x_{0}\right)$. Then, the true energy order of case 4 is two as we have predicted. Table 1 summarizes the energy orders of the $u$ 's for all four cases. The right arrow stands for suppressions or cancellations.

We can generalize the above argument to any other multi-tensor amplitudes as far as no other particle contains polarizations $e^{T_{i}}$. In order to make discussion simple, we choose the physical states for $V_{1}, V_{3}$ and $V_{4}$ in the DDF gauge. In this gauge, the polarizations of positive-norm states will consist of $e^{T}$ and $e^{(L-P)}$ only (e.g., Eqs. (35)-(39)). Consequently, suppressions which we have seen in the previous example do not happen and true leading amplitudes are simply given by the zeroth-order saddle-point approximation. In particular, we expect that $u_{\mathrm{I}}\left(x_{0}\right)$, analogously defined as in Eq. (47), should give the leading contribution to the high-energy amplitudes. Because $u_{\mathrm{I}}\left(x_{0}\right)$ depends on the polarization of $V_{2}$ only through factors of $e^{A} \cdot k_{13}$, its true energy order is exactly same as what we expect from the naive dimension of $V_{2}$. The only exception might happen if we consider amplitudes with subleading particles. For subleading particles in the DDF gauge, there are several terms which make the leading contributions to $u_{\mathrm{I}}\left(x_{0}\right)$, for example, $\alpha_{-2}^{T}|0, p\rangle$ and $\sqrt{2} \alpha_{-1}^{T} \alpha_{-1}^{(L-P)}|0, p\rangle$ in Eq. (36). If there is an unexpected cancellation among these terms, we cannot rely on the above argument and must judge the assumption on a case-by-case basis. However, cancellation itself is easily checked by calculating one factor of $u_{\mathrm{I}}\left(x_{0}\right)$, which is related to the polarization of the subleading particle. Therefore, as we did in the previous example, after checking that there is no such a cancellation, we can conclude that the assumption is valid. Thus, we can expect that our assumption is widely valid as far as the other particles do not contain polarizations of $e^{T_{i}}$.

On the other hand, if we consider amplitudes with $e^{T_{i}}$, our assumption easily breaks down. For example, if an amplitude contains one photon with the polarization $e^{T_{i}}$, the amplitude vanishes unless it has another particle with polarization $e^{T_{i}}$. Therefore, the leading particle should be $\partial X^{T_{i}}\left(\partial X^{T}\right)^{n-1} e^{i k \cdot X}$, not $\left(\partial X^{T}\right)^{n} e^{i k \cdot X}$. Thus, our assumption (7) is not valid. However, if we replace the state $|n\rangle$ by $|n-1\rangle^{\prime} \equiv \alpha_{-1}^{T_{i}}\left(\alpha_{-1}^{T}\right)^{n-1}|0\rangle$ and repeat the same argument in Section 2, one should be able to derive similar results based on this reference state.

\section{Acknowledgements}

The authors thank Masako Asano and Hiroyuki Hata for helpful discussions. This work is supported in part by the National Science Council, Taiwan, ROC and National Center for Theoretical Sciences, Hsinchu, Taiwan, ROC (grant NSC 94-2119-M-002-001).

\section{References}

[1] D.J. Gross, High energy symmetry of string theory, Phys. Rev. Lett. 60 (1988) 1229; D.J. Gross, Philos. Trans. R. Soc. London A 329 (1989) 401. 
[2] D.J. Gross, P. Mende, Phys. Lett. B 197 (1987) 129;

D.J. Gross, P. Mende, Nucl. Phys. B 303 (1988) 407;

D.J. Gross, J.L. Manes, The high energy behavior of open string theory, Nucl. Phys. B 326 (1989) 73.

[3] C.T. Chan, J.C. Lee, Stringy symmetries and their high-energy limits, Phys. Lett. B 611 (2005) 193, hep-th/0312226; C.T. Chan, J.C. Lee, Zero-norm states and high-energy symmetries of string theory, Nucl. Phys. B 690 (2004) 3, hep-th/0401133.

[4] C.T. Chan, P.M. Ho, J.C. Lee, Ward identities and high-energy scattering amplitudes in string theory, Nucl. Phys. B 708 (2005) 99, hep-th/0410194.

[5] C.T. Chan, P.M. Ho, J.C. Lee, S. Teraguchi, Y. Yang, Solving all 4-point correlation functions for bosonic open string theory in the high energy limit, hep-th/0504138.

[6] See, for instance, J. Isberg, U. Lindstrom, B. Sundborg, G. Theodoridis, Nucl. Phys. B 411 (1994) 122, hepth/9307108;

B. Sundborg, Nucl. Phys. B (Proc. Suppl.) 102 (2001) 113, hep-th/0103247;

E. Sezgin, P. Sundell, Nucl. Phys. B 644 (2002) 303, hep-th/0205131;

C.S. Chu, P.M. Ho, F.L. Lin, JHEP 0209 (2002) 003, hep-th/0205218;

G. Bonelli, Nucl. Phys. B 669 (2003) 159, hep-th/0305155.

[7] G.W. Moore, Finite in all directions, hep-th/9305139;

G.W. Moore, Symmetries of the bosonic string S-matrix, hep-th/9310026;

P.C. West, Physical states and string symmetries, Mod. Phys. Lett. A 10 (1995) 761, hep-th/9411029;

N. Moeller, P. West, hep-th/0507152;

J.C. Lee, Phys. Lett. B 241 (1990) 336;

J.C. Lee, Phys. Rev. Lett. 64 (1990) 1636;

J.C. Lee, B. Ovrut, Nucl. Phys. B 336 (1990) 222;

T.D. Chung, J.C. Lee, Phys. Lett. B 350 (1995) 22, hep-th/9412095;

T.D. Chung, J.C. Lee, Z. Phys. C 75 (1997) 555, hep-th/9505107.

[8] E. Del Giudice, P. Di Vecchia, S. Fubine, Ann. Phys. 70 (1972) 378;

R.C. Brower, P. Goddard, Nucl. Phys. B 40 (1972) 437;

R.C. Brower, Phys. Rev. D 6 (1972) 1655;

C.T. Chan, J.C. Lee, Y. Yang, Anatomy of zero-norm states in string theory, Phys. Rev. D 71 (2005) 086005, hepth/0501020. 